The Egyptian International Journal of Engineering Sciences \& Technology, Vol 3, No 1 (1999)

\title{
Filtering Digital Images Using Convolution
}

I.A ismaeil, MAHER HUSSEIN

\begin{abstract}
Image filtering is applied by using the convolution technique over some images

The filter used is the low-pass filter in the carbox form. Artificially added noise to some

images are filtered and the pixels obtained are augmented using 2D-interpolation method

Results are very acceptable
\end{abstract}

